\title{
Um Modelo para Detecção de Ansiedade Computacional em Idosos Interagindo com a Web
}

\author{
Thiago Donizetti dos Santos \\ Universidade Federal do ABC \\ Santo André, SP, Brasil \\ thiagods05@gmail.com
}

\author{
Vagner Figueredo de Santana \\ IBM Research \\ São Paulo, SP, Brasil \\ vagsant@br.ibm.com
}

\section{RESUMO}

Com o aumento do uso de diferentes tipos de tecnologias no cotidiano, seria esperado que usar computadores fosse confortável para todos. Porém, algumas pessoas ainda sentem-se apreensivas antes e durante o uso de computadores, enfrentando múltiplos níveis de ansiedade e, para níveis elevados, demonstram o que é chamado de Ansiedade Computacional (do inglês CA, Computer Anxiety). Pessoas com CA enfrentam problemas no uso de tecnologias em vários contextos, incluindo situações nas quais utilizam computadores por não terem alternativa, tais como ao utilizar serviços governamentais disponíveis somente por meio de sistemas computacionais, exercer a cidadania votando com urnas eletrônicas, caixas eletrônicos e ao utilizar computadores no trabalho. A CA pode criar múltiplas barreiras para essas pessoas, privandoas do acesso à tecnologia, à educação, ao pleno exercício da cidadania, entre outros. Neste contexto, o presente projeto investigou as principais características relacionadas a como idosos com CA interagem com computadores e como essas características são representadas em logs de interação. Para tal, realizou-se um estudo de campo com 31 participantes idosos envolvendo captura de dados de interação, dados de rastreamento ocular, escalas de rastreamento de níveis de depressão, ansiedade, ansiedade computacional, entre outras. Com os resultados, um modelo para detecção de CA foi obtido. Tal modelo é capaz de identificar diferentes níveis de CA (i.e., sem ansiedade, baixo nível de CA e alto nível de CA) com $83,87 \%$ de acurácia, mostrando que é possível identificar diferentes níveis de $\mathrm{CA}$ de idosos interagindo com a Web analisando logs de interação.

Permission to reproduce or distribute, in whole or in part, material extracted from this work, verbatim, adapted or remixed, as well as the creation or production from the content of such work, is granted without fee for noncommercial use, provided that the original work is properly credited.

IHC 2019 - TRILHA ARTIGOS INTERNACIONAIS, Outubro 21-25, 2019, Vitória, Brasil. In Anais Estendidos do XVIII Simpósio Brasileiro sobre Fatores Humanos em Sistemas Computacionais. Porto Alegre: SBC

(C) 2019 by the author(s), in accordance with the terms of the Creative Commons Attribution-NonCommercial 4.0 International Public License (CC BY-NC 4.0).

\section{PALAVRAS-CHAVE}

Ansiedade Computacional, Envelhecimento, Idosos, Accessibilidade, Usabilidade, Experiência de Usuário, UX.

\section{INTRODUÇÃO}

Atualmente, diversos serviços estão disponíveis na internet. Escolas e faculdades oferecem cursos à distância em ambientes virtuais de aprendizagem e sistemas governamentais fornecem serviços tais como emissão de documentos, agendamento de atendimento e declaração de imposto de renda. A oferta de serviços eletronicamente visa desburocratizar e agilizar o acesso. Porém, o fato de só estarem disponíveis por meio de computadores pode criar barreiras para pessoas que enfrentam problemas no uso de computadores, que podem ocorrer por falta de experiência, por não saberem como operá-los, por não terem acesso a eles e, em alguns casos, podem ter medo de danificá-los ou de não conseguir realizar tarefas corretamente. Essas pessoas podem enfrentar múltiplos níveis de ansiedade e, para níveis elevados, demonstram o que é chamado de Ansiedade Computacional (CA, do inglês Computer Anxiety). Estudos sobre CA encontrados em [4], mostram que a CA está relacionada à falta de usabilidade e acessibilidade nos sistemas. Mostram também a forte correlação (inversa) entre CA e Autoeficácia Computacional, i.e., pessoas com baixa autoeficácia tendem a ter maiores níveis de $\mathrm{CA}$ e vice versa. Em relação às pessoas com $\mathrm{CA}$, os estudos mostram que os idosos são os que mais apresentam CA, devido aos problemas para se manterem atualizados em relação às novas tecnologias, por não usarem computadores desde a infância e por causa dos esteriótipos relacionados ao uso de tecnologia por idosos.

\section{MÉTODO}

Foi realizado um estudo de campo com 31 participantes idosos com o objetivo de identificar sinais de CA utilizando a análise de logs de interação, capturados durante a interação do usuário com a Web. A captura foi realizada via plugin [3] instalado no navegador. Os dados capturados foram analisados e modelos computacionais para detecção de CA foram produzidos utilizando algoritmos de classificação supervisionada. 
Para criar os modelos, todos os algoritmos de classificação supervisionada do Weka (https://www.cs.waikato.ac.nz/ml/weka/) foram testados. $\mathrm{O}$ algoritmo que obteve melhor acurácia foi o Random Tree, em concordância com resultados presentes na literatura para problemas de classificação utilizando dados de logs de interação [1].

\section{RESULTADOS}

A partir da seleção do algoritmo, diferentes atributos foram considerados, representando diferentes cenários, disponibilidade de escalas, sensores e dados demográficos, resultando em três diferentes modelos computacionais. O modelo que obteve o melhor resultado (Figure 1) foi criado utilizando as métricas referentes aos dados capturados pelo plugin utilizado. As métricas utilizadas envolvem uso do mouse (e.g., distância percorrida, velocidade, número de cliques e pausa antes de cliques), métricas obtidas a partir do grafo de uso (e.g., total de eventos disparados, picos de excentricidade e grau médio) e tempo que o participante levou para realizar a tarefa.

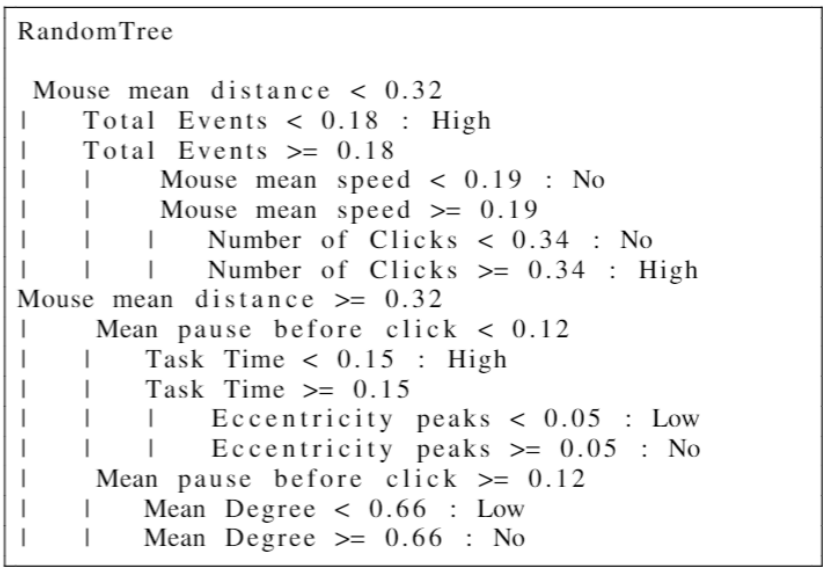

Figura 1: Modelo computacional obtido com melhor acurácia. No, low e high representam, respectivamente, os grupos sem CA, CA moderada e CA elevada. Dessa forma, as folhas representam as classes consideradas pelo algoritmo e os nós intermediários as métricas de interação consideradas pelo modelo.

\section{CONCLUSÃO}

O uso de algoritmos de classificação baseados em árvores de decisão, como o Random Tree, traz vantagens em relação à explicabilidade se comparado a outros algoritmos de classificação. O Random Tree gera uma árvore que permite identificar as métricas utilizadas e a influência delas na classificação. Tal explicabilidade é um aspecto fundamental para a área de Interação-Humano Computador (IHC), pois permite relacionar o modelo resultante com as variáveis presentes na Outra vantagem importante de algoritmos baseados em árvore é o fato de serem de fácil implementação e eficientes, permitindo a execução em tempo real durante a interação do usuário com o sistema.

Acredita-se que este trabalho é relevante para a comunidade de IHC no Brasil por se tratar de um estudo abordando um problema atual, enfrentado não só por idosos mas também por pessoas com pouca experiência com computadores: a Ansiedade Computacional. A CA tem impacto direto no desempenho, na acessibilidade e na usabilidade percebida pelo usuário, uma vez que influencia a maneira como ele percebe e interage com os computadores.

A principal contribuição deste trabalho é um modelo computacional capaz de detectar a CA a partir da análise de logs de interação, possíveis de se capturar utilizando um data logger. Os dados utilizados no modelo podem ser capturados durante qualquer interação envolvendo um usuário e uma página Web, tornando possível o uso deste modelo para capturar e analisar dados, identificando usuários com sinais de CA em tempo real, podendo usar esta informação para dar suporte ou personalizar a interface de usuário. Por exemplo em sistemas governamentais que são muito úteis para a população, mas muitas vezes causam dúvidas quanto sua utilização, fazendo com o que as pessoas precisem de ajuda de terceiros para utilizá-los de forma correta [2].

Por fim, a apresentação do trabalho pode ser uma oportunidade de obter feedback sobre o trabalho e sugestões para sua continuidade, já que ele faz parte de um projeto maior que visa, não só a identificação de usuários com $\mathrm{CA}$, mas também de elementos de interface de usuário relacionados a sintomas de CA.

\section{REFERÊNCIAS}

[1] Fabiano M. de Moraes, Vagner F. de Santana, and Juliana C. Braga. 2016. Supporting the selection of web content modality based on user interactions logs. In Proceedings of the 13th Web for All Conference. ACM, 40.

[2] Chee W. Phang, Juliana Sutanto, Atreyi Kankanhalli, Yan Li, Bernard C. Tan, and Hock-Hai Teo. 2006. Senior citizens' acceptance of information systems: A study in the context of e-government services. IEEE Transactions on Engineering Management 53, 4 (2006), 555-569.

[3] Vagner F. de Santana and Felipe E. Silva. 2019. User Test Logger: An Open Source Browser Plugin for Logging and Reporting Local User Studies. Proceedings of HCI International 2019 (2019).

[4] Thiago D. dos Santos and Vagner F. de Santana. 2018. Computer Anxiety and Interaction: A Systematic Review. In Proceedings of the Internet of Accessible Things. ACM, 18. 